\section{European Who's Who in Condensed Matter Physics}

The first edition of the European Who's Who in Condensed Matter Physics aimed at facilitating communication between physicists working in the field of condensed matter is in press. The compendium will help the condensed matter scientist, and particularly the young researcher and the graduate student, to establish contacts with other individuals and institutions and to collect information on activities in a given condensed matter area pursued in Europe. The European Who's Who in CMP is also intended for organizational work, e.g. for the compilation of mailing lists of workshops and conferences and any similar purpose.

The large amount of data is contained in two lists:

In the main list the institutions with the individuals affiliated to them are given in alphabetical order of the countries and - within a given country - in numerical or alphanumerical order of the postal code. Both for significant subdivisions of an institute and for the individuals the main fields of activity are specified in terms of PACS indices. For Individual Ordinary Members of EPS also the appropriate Section within the Condensed Matter Division is given.

The second list contains the names of all individuals listed in alphabetical order throughout Europe. For each entry the institute code is given for cross reference.

In total, the book contains about 2500 names and addresses of European physicists working in the field of condensed matter and close to 500 institutions. It has been produced from camera-ready laser printed originals and will be ready in the early Autumn. The copyright belongs to the European Physical Society. Copies can be ordered now, in advance, from EPS by transferring:

SFR 15. - per copy for Individual Ordinary Members

SFR 20. - per copy for other EPS members and non-commercial institutions

SFR 200. - per copy for commercial institutions

to the following bank account: Swiss Bank Corporation

164.899 (EPS)

$\mathrm{CH}-1211$ Geneva 11

Payment by credit card or Eurocheque cannot be accepted.

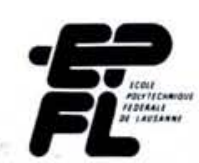

\section{Swiss Federal Institute of Technology Lausanne (Ecole Polytechnique Fédérale de Lausanne)}

announces several openings for the position of

\section{full professor in experimental physics}

in the following domains:

- Physics of electronic materials

- Physics of semicrystalline solids

- Surface physics / Surface science

- Physics for biomedical engineering

Teaching is given in French.

Applications deadline:

November 30th, 1989.

The successful candidates are expected to start their activities in summerfall 1990.

For more information, and application, write to:

Secrétariat général de l'Ecole Polytechnique Fédérale de Lausanne. CE-Ecublens,

CH-1015 Lausanne, Switzerland.

\section{Solar Newsletter}

The Solar Physics Section of the Astronomy and Astrophysics Division has published the first issue of its planned bi-annual Newsletter. Editor is R.J. Rutten of the Sterrekundig Instituut in Utrecht. To receive the Newsletter it is essential to make application to:

G.W. Geijtenbeck, Administrator ESPN

Sterrekundig Instituut

Postbus 8000

NL - 3508 TA Utrecht

even if you are already a member of the Section. Forms are available for this from Utrecht or from the EPS Secretariat. Contributors should send their material (preferably in the form of a Latex email file) to the Editor at the above address.

Tel. (30) 535200 Fax (30) 531601

\section{LEP Operational}

By 14 August, just one month after the first circulation of a beam in CERN's electron-positron collider LEP, $Z^{\circ}$ particles had been identified in all four of the detectors set up at the intersection points (see Europhysics News, June 1989). Beam tuning and refining of the systems will have priority before data taking begins in earnest in the Autumn.

\title{
Cooperation between EPS and EMRS
}

The European Materials Research Society was founded in 1983 as a multidisciplinary association of scientists and engineers to promote efforts and advances in materials research and new technologies within Europe. To enhance technology transfer, EMRS organizes and sponsors conferences, workshops, courses and summer schools, meetings and seminars. Major conferences are held once or twice a year at the Council of Europe building in Strasbourg. Each of these conferences comprises three or four independently organized symposia running over the entire length of the conference.

Since the foundation of the EMRS there have been close links with EPS and in particular with its Condensed Matter Division (CMD) in order to exchange information about scientific activities and conference planning. Recently, EMRS and the CMD have decided to enter a more active form of interaction by co-organizing part of their respective conferences.

To begin with, one of the symposia of the June 1990 (29 May - 1 June) EMRS Conference in Strasbourg, namely the symposium on "Superconductors", will be co-organized with the CMD which has nominated Y. Bruynseraede and G. Deutscher to act for it. Under the same token, the CMD has set aside a considerable part of the programme of its 10th General Conference to be held in Lisbon, Portugal, 9-12 April 1990. The section run under the heading "Physics of Materials for Future Electronics" will be co-organized with the EMRS which has nominated P. Glasow and A. Golanski for the purpose.

Both projects are considered to be the encouraging beginning of a fruitful cooperation to the benefit of European science and European scientists. Many condensed matter physicists consider themselves to be also materials researchers and vice versa; the area of overlap and the amount of common interest are immense. It is only a logical consequence that the two organizations move closer together and it is hoped to enlarge their joint activities in future years. But first comes the year 1990 when both Lisbon and Strasbourg welcome you. Put the dates in your memory file straight away.

G. Harbeke \& M. Rodot 